Abstract

\title{
Prevalence of Malnutrition and Dysphagia in Advanced Age Adults Newly Admitted to Age-Related Residential Care ${ }^{\dagger}$
}

\author{
Stacey Senior ${ }^{1}$, Marilize Richter ${ }^{2}$, Jacqui Allen ${ }^{2}$ and Carol Wham 1,* \\ 1 School of Sport, Exercise and Nutrition, Massey University, Auckland 0742, New Zealand; \\ stacey.senior05@gmail.com \\ 2 Department of Surgery, University of Auckland, Auckland 1023, New Zealand; \\ M.Richter@massey.ac.nz (M.R.); jeallen@voiceandswallow.co.nz (J.A.) \\ * correspondence: C.A.Wham@massey.ac.nz \\ + Presented at the 2018 Nutrition Society of New Zealand Annual Conference, Auckland, New Zealand, \\ 28-30 November 2018.
}

Published: 7 March 2019

Background: Adults over 80 years are the fastest growing population segment predicted to increase six-fold by 2050. Older people admitted to age-related residential care (ARRC) maybe nutritionally vulnerable as the result of suboptimal food intake as a secondary consequence of comorbidities, increased functional difficulties and cognitive decline. The aim of this study was to establish the prevalence of nutrition risk and associated risk factors among adults of advanced age recently admitted to ARRC.

Methods: A cross-sectional study was conducted among participants aged $\geq 85$ years within five days of admission to ARRC within the Waitemata District Health Board. Sociodemographic and health characteristics of the participants were ascertained during personal interviews. Body composition, grip strength and gait speed were recorded using standardised measures. Nutrition risk was determined using the Mini Nutritional Assessment-Short Form (MNA-SF), dysphagia risk using the 10-Item Eating Assessment Tool (EAT-10) and cognitive status using the Montreal Cognitive Assessment (MoCA).

Results: Among 97 participants (mean age $90.9 \pm 3.8$ years), half (50.5\%) were malnourished, $40.2 \%$ were at nutrition risk and a third (37.1\%) were at risk of dysphagia. Malnourished vs. wellnourished/at risk participants were more likely to be $\geq 90$ years $(p=0.019)$, admitted to ARRC on a permanent basis $(p=0.016)$, at dysphagia risk $(p=0.015)$, have a BMI $<23(p=0.022)$, lower fat mass $(p=0.005)$, and fewer comorbidities $(p=0.030)$. The MNA-SF score was inversely correlated with age $(r=-0.225, p=0.027)$ and positively correlated with BMI $(r=0.499, p=<0.001)$ and fat mass $(r=0.765$, $p=<0.001)$.

Conclusions: Malnutrition and dysphagia risk were prevalent among advanced age adults, especially among those $\geq 90$ years with a low BMI who are an easily identifiable group. Early screening and intervention is critical upon admission to ARRC. 